\title{
A simple and accurate dosimetry protocol to estimate activity for hyperthyroidism
} treatment

\author{
Andrea Skanjeti ${ }^{1,2}$, Anna Miranti ${ }^{3}$, Gabriela Mariam Delgado Yabar ${ }^{1}$, Davide Bianciotto ${ }^{1}, E_{d o a r d o}$ Trevisiol $^{1}$, Michele Stasi ${ }^{3}$, \\ Valerio Podio' \\ ${ }^{1}$ Nuclear Medicine Unit, San Luigi Gonzaga Hospital, University of Turin, Orbassano, Italy \\ ${ }^{2}$ Medical Science Department, University A. Avogadro, Novara, Italy \\ ${ }^{3}$ Medical Physics Department, IRCC Candiolo, Turin, Italy \\ Financial support was not required nor obtained. \\ The authors declare also that they have no conflict of interest.
}

[Received 20 V 2014; Accepted 19 XII 2014]

\begin{abstract}
BACKGROUND: Aim of the study was to evaluate accuracy of different dosimetry protocols in estimating the required ${ }^{131}$ | activity to treat hyperthyroid patients.

MATERIALS AND METHODS: Forty consecutive patients were analysed: twenty-eight Graves' disease; twelve autonomous thyroid nodule (ATN). Maximum-uptake, effective half-time and residence-time were estimated from Radioiodine Uptake Test. Residence-time was estimated using a bi-compartmental model. For ${ }^{131}$ I activity calculation, algorithms laid down in European Association of Nuclear Medicine (EANM) guidelines, ICRP 53 approach and a mono-exponential formula (ME), were compared with OLINDA/EXM results.

RESULTS: Based on EANM guidelines, activities to be administered were $3 \%$ higher in Graves' disease ( $p=0.001)$ and $3 \%$ higher in ATN $(p=0.046)$. Calculated activities using ICRP 53 approach were significantly lower compared to OLINDA/EXM: 33\% in Graves' disease; $17 \%$ in ATN. Activities recommended by ME, were significantly higher: in Graves' disease $20 \%$; $42 \%$ in ATN. CONCLUSIONS: Only EANM algorithm predict quite well, compared to OLINDA/EXM, the required activity to treat hyperthyroid patients.
\end{abstract}

KEY words: Graves' disease, autonomous thyroid nodule, radioiodine, MIRD, guidelines

Nuclear Med Rev 2015; 18, 1: 13-18

\section{Background}

Radioiodine ( $\left.{ }^{131} \mathrm{I}\right)$ has been successfully used to treat hyperthyroidism ever since Hertz and Roberts used it for the first time on humans. This was spring 1941 [1]. Testament to the safety, efficacy and convenience of this radiopharmaceutical is the fact that it has now become the first-line treatment for hyperthyroidism [2-4]. Ever since the start, the activity rate most conducive to optimal treatment aroused as a matter of debate: seventy years down the line, the clarification of this keeps eluding researchers and physicians alike [5]. Some authors advocate administering a fixed activity regimen. In addition to its straightforward applicability, this method

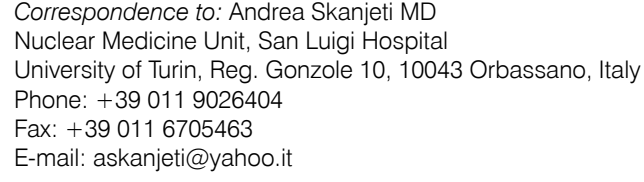

presents another advantage: namely, in case of treatment failure, ${ }^{131}$ I can be redelivered, whereas in case of hypothyroidism, life-long treatment with levothyroxine remains a safe option [3, 6]. Other authors recommend, instead, using a patient-specific dosimetric method based on calculated activities [3]. This is also the approach set forth in institutional guidelines, such as the Euratom Directive 43/97 [7], the ICRU (International Commission on Radiological Units and Measurements) Report 67 [8], as well as in the French, German and Italian legislations [9-11]. Furthermore, several papers have reported a dose-related outcome of radioiodine treatment $[1,12,13]$. Positive correlation has been identified between hypothyroidism and radiation dose, while the irradiation of non-target tissues has also emerged as a factor that needs careful consideration [14]. Several studies have concentrated on autonomous thyroid nodules (ATN), highlighting significant post-treatment rates of hypothyroidism: still a rather unsatisfactory outcome after seventy years of research. Focusing on the dose received by healthy thyroid tissues, some authors have found that both lobes — ipsilateral and 
contralateral - absorbed more than one tenth of the node dose $[15,16]$. This unnecessary irradiation could perhaps be responsible for the ensuing hypothyroidism. Moreover, roughly $15 \%$ of hyperthyroid patients have accelerated radioiodine turnover within the thyroid gland [17, 18], an outcome attributed to a small thyroid iodine pool (small-pool syndrome). This can result in higher than expected absorbed doses to the extra-thyroid organs and tissues, and particularly to the bone marrow and the blood pool $[17,18]$. For these reasons, we share with the above-mentioned authors and institutions the view that every effort should be made in order to keep the irradiation of no-target tissues to the minimum. Once the target organ dose has been decided on, a number of methods can be applied to estimate the amount of activity required for planned dose delivery to the thyroid gland $[9,19]$. The Committee on Medical Internal Radiation Dose (MIRD) is credited with developing a standardised data framework to establish correlations between delivered activity and radiation dose [20]. The effectiveness of the MIRD approach has been widely discussed and proved excellent even compared to more sophisticated methods, such as the Monte Carlo calculations [21-23]. OLINDA/EXM is a recent tool, which implements the MIRD standard phantom-based methodology developed for dosimetric purposes [24, 25]. However, institutions such as the International Commission on Radiological Protection (ICRP), the European Association of Nuclear Medicine and Molecular Imaging (EANM), as well as other authors have proposed different formulae, based on MIRD formalism, in order to estimate the amount of ${ }^{131}$ I activity needed to reach the planned dose $[9,19,26]$. These approaches, which basically simplify biokinetic data, have been already used, or advised for use, in nuclear medicine routine as well as in research studies [27-29] because they do not require any specially engineered device for their application.

The aim of this study is to evaluate, by using a comparison with the results of the OLINDA/EXM software, the European guidelines, ICRP approach as well as a simplified formula derived by the MIRD formalism, based on a mono-exponential function of radiodiodine kinetics (the latter was used by the old Italian guidelines [9]) for estimating the amount of ${ }^{131}$ activity to be administered to patients with either Graves' disease or ATN

\section{Materials and methods}

\section{Patient selection}

Forty consecutive patients, with either Graves' disease $(n=28)$ or ATN $(n=12)$, were included in this explanatory work. All were referred to our centre for radioiodine treatment and were suspected for fast to very fast iodine metabolism (small-pool syndrome); none of the patients was in relapse. Thyrotoxicosis was diagnosed on clinical and laboratory findings (high thyroxine, low thyroid-stimulating hormone).

Prior to radioiodine, all patients underwent planar pertechnetate scintigraphy and ultrasonography. Following these, either Graves' disease or ATN was diagnosed. With reference to ATN patients, no significant uptake was observed in the rest of the gland, i.e. the tracer was concentrated within the node. Given their intrinsic ability to show the presence of functional tissue, scintigraphic images were also used for thyroid mass estimation [30], assuming a density of $1 \mathrm{~g} / \mathrm{cm}^{3}$.

Based on the European guidelines [19], all patients were instructed to suspend anti-thyroid treatment (methimazole or propylthiouracil) at least 10 days before the radioiodine uptake test and at least 20 days before radioiodine treatment. The study was carried out in accordance with the ethical guidelines of the local Ethics Committee for Clinical Investigation. All patients gave their informed written consent prior to the Radioiodine Uptake Test (RUT).

\section{Radioiodine uptake test}

Radioiodine uptake test was performed according to standard operational procedures for pre-therapeutic dosimetry prior to the treatment of benign thyroid diseases [27]. Each patient received an orally administered capsule containing $1-3 \mathrm{MBq}$ of [ $\left.{ }^{131} \mathrm{I}\right]$ lodide, which was measured at delivery $(t=0)$. Because these patients, based on prior information such as clinical and scintigraphic data, were suspected to have a fast iodine metabolism, uptake measurements were performed each hour in the first ten hours and then at $24,25,26,27,28,48,72$ and 96 hours. This approach was studied for a correct assessment of iodine uptake in the first phase and of iodine dismissing in the second phase. Measurements were performed with a 2-inch $\mathrm{Nal}(\mathrm{TI})$ detector at a 25-centimeter distance between detector and source (capsule or thyroid). The energy window was set at 328-400 keV. All data were corrected for background (patient and room, respectively). Three 60-second acquisitions were performed for each session and the average was used for successive analysis. To ensure optimal reproducibility, all sessions of each patient were performed by a single technologist (G.M.D.Y or D.B.). Residence time and effective half time were calculated with reference to the ratio between each measurement and the measurement at $t=0$ of the capsule containing the administered activities, as prescribed by official European guidelines [19].

\section{Algorithms and methods used to calculate the activity required to deliver a determined radiation dose}

In order to estimate the activity necessary to deliver the planned dose to thyroid gland or ATN nodule, both OLINDA/EXM and the EANM guidelines require knowledge of radioiodine residence time within the target tissue. Residence time was calculated based on RUT measurements and is equal to the area under the RUT measurements-time curve (integral from 0 to infinity of the function used to fit the RUT data):

$$
\frac{U_{p(t)}}{U_{c(0)}}=\left(e^{-a t}-e^{-b i}\right) \cdot \frac{a \cdot U}{(b-a)}
$$

where $\frac{U_{p(t)}}{U_{c(0)}}$ is patients' thyroid uptake at time $t$, normalized for capsule uptake at time $0 ; U$, $a$ and $b$ were estimated with reference to the function fit and represents radioiodine transfer rate (a: elimination from thyroid; b: elimination from blood; $U$ a : thyroid uptake). We chose this function taking into account ${ }^{131}$ alleged bi-compartmental kinetics (thyroid and blood pool) in benign thyroid diseases [10, 11, 27, 31]. In all cases the fit was optimal, $R^{2}$ was higher than 0.90 . The residence time (RT) is therefore:

$$
R T=\left(\frac{1}{a}-\frac{1}{b}\right) \cdot \frac{a \cdot U}{(b-a)}
$$

Radioiodine activity to be administered was estimated by the mean of OLINDA/EXM given the planned dose and according the respective phantom (male/female); subsequently it was corrected to match the real size of the gland or nodule. 
Table 1. Data of thyroid or nodule mass, maximum uptake, target dose, effective half time and residence time for Graves's disease and autonomous thyroid nodule

\begin{tabular}{lcccccc} 
& Mass $[\mathrm{g}]$ & $\mathrm{U}_{\max }$ & Target Dose (Gy) & $\mathrm{T}_{1 / 2 \mathrm{fff}}$ [h] & Res Time $[\mathrm{h}]$ \\
\hline Graves' (Mean $\pm \mathrm{SD}$ ) & $30.7 \pm 15.8$ & $0.51 \pm 0.13$ & 200 & $111.1 \pm 38.3$ & $96.9 \pm 34.3$ \\
ATN (Mean $\pm \mathrm{SD}$ ) & $11.3 \pm 10.3$ & $0.28 \pm 0.05$ & 300 & $111.7 \pm 26.9$ & $58.6 \pm 14.1$
\end{tabular}

ATN — autonomous thyroid nodule, SD — standard deviation, Max up — maximal uptake, $T_{1 / 2 \text { eff }}(h)$ - effective half time, Res Time — Residence time, $g$ - gram, Gy — Gray, $h$ - hour

To evaluate the radioiodine dose to ATN, we used the OLINDA/EXM Unit Density Sphere Model, in order to better approximate the reality, given that the dosimetric calculations becomes highly geometry-dependent in small structures [32]. Entering residence time data, a list of dose coefficients, expressed in mGy/MBq or $\mathrm{mG} / \mathrm{mCi}$, was outputted by OLINDA/EXM. These coefficients are reported for different masses of the nodule, in a range of masses between 0.01 and $6,000 \mathrm{~g}$ and in different steps (of $2 \mathrm{~g}$ in the range $2-10 \mathrm{~g}$ and in steps of $20 \mathrm{~g}$ in the range $20-100 \mathrm{~g}$ ).

Dose coefficients were fitted very well $\left(R^{2}>0.999\right)$ according to the following power function

$$
A T N_{\text {casse cueff. }}=j \cdot \text { nodule mass }{ }^{k}
$$

$k$ and $j$ values were obtained from the fit in order to obtain dose coefficients for the real mass of the patient's nodule.

Although based on the MIRD S-value, the EANM guidelines, as well as the subsequent standard operational procedures, indicate the following algorithm for activity calculations:

$$
A_{\text {LEAMM }}=\frac{F}{\ln 2} \cdot\left(\frac{D \cdot m}{R T}\right)
$$

where $A_{\text {EANM }}[M B q]$ is the activity to be administered to deliver a target dose $D$ [Gy], $m$ is the thyroid mass [g], while $F$ is a constant derived from $\mathrm{S}$-values and is equal to

$$
24.7 \% \frac{M B q \cdot d w y}{g \cdot G y} \quad[4,19,20,27] .
$$

Finally, in order to analyse the required activity independently from both the dose and the thyroid mass, the calculated activity normalized by the ratio of the dose to the mass, $A / D / m$, was considered.

ICRP 53 does not suggest any algorithm for the calculation of the activity required to deliver a planned radiation dose to thyroid gland or ATN tissues, but proposes a conversion factor for six different maximal uptakes of the $20 \mathrm{~g}$ thyroid reference mass (5\%, 15\%, 25\%, $35 \%, 45 \%, 55 \%)$. A linear function was fitted from these data, and the activity was then normalized to the mass of the patient's thyroid as previously reported in a paper which used this protocol to calculate the activity to be administered in hyperthyroid patients [28].

The last algorithm, used in this study even if it is based on MIRD formalism, is certainly easier to use given that it does not require residence time. This dosimetric protocol has been used in various papers [29]; it describes the radioiodine kinetics considering a Mono-Exponential (ME) function and it was indicated in the former Italian guidelines [9]:

$$
A_{M:}=5.829 \cdot \frac{D \cdot m}{U_{\max } \cdot T_{1 / 2} \text { eff }}
$$

where $A_{M E}[M B q]$ is the activity to be administered to deliver a target dose $D[G y], m$ is the thyroid mass $[g], U_{\max }$ is the maximal uptake value during RUT and $T_{1 / 2 \text { eff }}[\mathrm{h}]$ is the effective radioiodine half-time during the dismissing phase estimated by logarithmic function based on at least three RUT measurements.

\section{Statistical analysis}

In order to compare results obtained by using the various activity calculation formulas, we used Student's paired $t$ test as appropriate. All statistically significant levels were set at $5 \%(p<0.05)$. SPSS vers. 19 (IBM Corporation, Armonk, NY, USA) was used for fitting purposes.

\section{Results}

\section{Thyroid parameters}

The mean thyroid/autonomous masses, maximum uptake, target doses, effective half-lives and residence times for Graves' disease and ATN, respectively, are illustrated in Table 1. Interestingly, the half-life data confirm, although not for all patients, our suspect of patients with fast iodine metabolism; their iodine biologic half-life was roughly 10 days [17].

\section{Different approaches to determine activities to be administered}

OLINDA/EXM calculated activities were used as gold standard. Based on the software calculations, we estimated that for patients with Graves' disease the activities needed to achieve the target doses were $644 \pm 259 \mathrm{MBq}$. A/D/m was $0.11 \pm 0.08 \mathrm{MBq} /$ $/$ Gy/g. For ATN patients, the required activities were $477 \pm 367 \mathrm{MBq}$, while $\mathrm{A} / \mathrm{D} / \mathrm{m}$ was $0.15 \pm 0.04 \mathrm{MBq} / \mathrm{Gy} / \mathrm{g}$.

Mean activities, as well as $A / D / m$ calculations based on the EANM algorithm, on the ICRP 53 approach and ME formula, are detailed in Table 2 for Graves' disease and ATN, while the individual values are presented in Figures 1 and 2, respectively.

With reference to the EANM algorithm, the activities to be administered and $\mathrm{A} / \mathrm{D} / \mathrm{m}$ were quite similar ( $+3 \%$ in both Graves' disease and ATN). In fact, this applies from a practical point of view only, while on statistical point of view the respective values were significantly different, due to small, homogeneous differences in the algorithm as compared to the reference method. Conversely, with reference to the ICRP 53 approach, the activities to be administered and $A / D / m$ were much lower both in Graves' disease and in ATN (33\% Graves' disease and 17\% in ATN). Finally, with reference to the $\mathrm{ME}$ dosimetric protocol, the required activities and A/D/m were significantly higher both in Graves' disease and in ATN patients (20\% in Graves' disease and $42 \%$ in ATN).

\section{Discussion}

We worked to fulfil ALARA Principle, international rules and national Laws (e.g. in France, in Germany, in Italy). Hyperthyroidism 
Table 2. Activities and activities per unit of Gy and g computed according to each approach (EANM Guidelines algorithm, ICRP 53 approach and the ME formula) and their differences with OLINDA/EXM

\begin{tabular}{|c|c|c|c|c|c|}
\hline \multirow[t]{2}{*}{ Approach } & \multirow[t]{2}{*}{ Pathology } & \multicolumn{2}{|c|}{ Graves' Disease } & \multicolumn{2}{|c|}{ ATN } \\
\hline & & Activity (MBq) & $\mathrm{A} / \mathrm{D} / \mathrm{m}(\mathrm{MBq} / \mathrm{Gy} / \mathrm{g})$ & Activity (MBq) & $\mathrm{A} / \mathrm{D} / \mathrm{m}(\mathrm{MBq} / \mathrm{Gy} / \mathrm{g})$ \\
\hline OLINDA/EXM & Mean \pm SD & $644 \pm 583$ & $0.11 \pm 0.08$ & $477 \pm 367$ & $0.15 \pm 0.04$ \\
\hline \multirow[t]{3}{*}{ ME } & Mean $\pm \mathrm{SD}$ & $774 \pm 667$ & $0.13 \pm 0.08$ & $678 \pm 606$ & $0.20 \pm 0.04$ \\
\hline & Differences with & $+20 \%$ & $+20 \%$ & $+42 \%$ & $+31 \%$ \\
\hline & OLINDA/EXM & & & & \\
\hline \multirow[t]{4}{*}{ ICRP 53} & Mean \pm SD & $431 \pm 230$ & $0.07 \pm 0.02$ & $398 \pm 321$ & $0.13 \pm 0.03$ \\
\hline & Differences with & $-33 \%$ & $-31 \%$ & $-17 \%$ & $-15 \%$ \\
\hline & OLINDA/EXM & & & & \\
\hline & p-value & 0.017 & 0.023 & 0.014 & 0.012 \\
\hline \multirow[t]{3}{*}{ EANM GL } & Mean \pm SD & $662 \pm 609$ & $0.11 \pm 0.08$ & $493 \pm 397$ & $0.15 \pm 0.04$ \\
\hline & Differences with & $+3 \%$ & $+2 \%$ & $+3 \%$ & $+1 \%$ \\
\hline & $\mathrm{p}$-value & 0.001 & $<0.0001$ & 0.046 & 0.024 \\
\hline
\end{tabular}

GL - guidelines, MBq - Megabecquerel, Gy — Gray, g - gram, SD — standard Deviation, Max up — maximal uptake, $T_{1 / 2 \text { eff }}(h)$ - effective half time

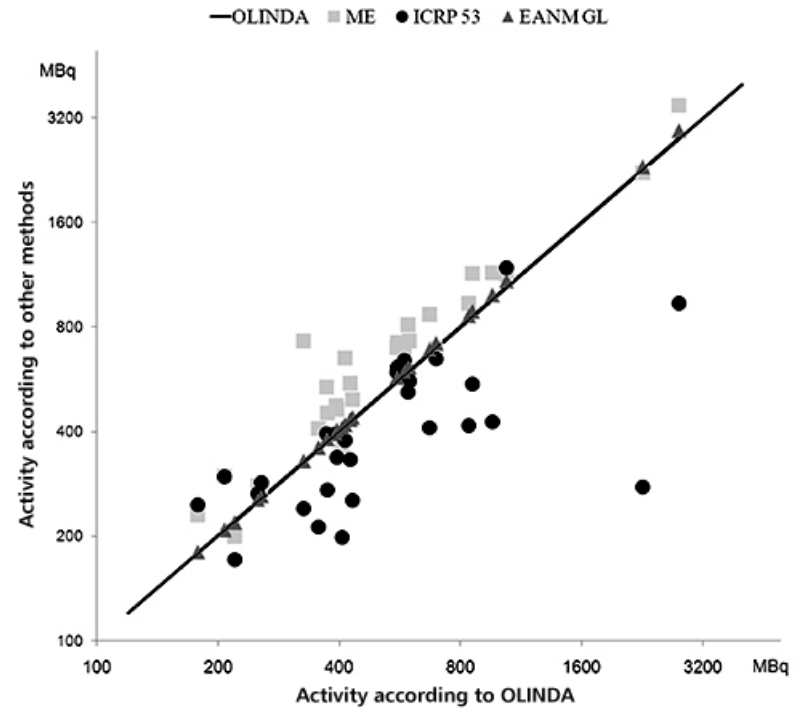

Figure 1. The graph shows computed activities, for each patient with Graves' disease, according three methods (triangles for EANM Guidelines algorithm, circles for ICRP 53 approach and squares for the ME formula). The black line presents the identity-line, i.e. values from OLINDA/EXM. Logarithmic scale was used for both axis for visual clarity purposes

must be eradicated by employing an individualized activity and the lowest dose [8-11]; this was the cornerstone on which was based this work.

Several issues are addressed in this paper: first, the compromise between treatment efficacy and reduction of doses to the blood pool/bone marrow (in Graves' disease patients) or to the normal tissues of the thyroid (in ATN patients) is intensely debated in the scientific community $[14,16,33]$. This study showed the importance of an accurate knowledge of kinetic data in calculation of radioiodine activity to be administered in hyperthyroid patients. In fact, the estimation of the biodistribution by the mean of residence time is more accurate than the assessment with estimated effective half-life (such as according to ME) or fixed half-life (such as accord-



Figure 2. The graph shows computed activities, for each patient with ATN, according three methods (triangles for EANM Guidelines algorithm, circles for ICRP 53 approach and squares for the ME formula). The black line presents the identity-line, i.e. values from OLINDA/EXM. Logarithmic scale was used for both axis for visual clarity purposes

ing to ICRP 53). Residence time is, in our view, a key factor, which estimation is required by the OLINDA/EXM software. We sampled frequently thyroid uptake (18 measurements for each of the 40 patients), even though we are aware that only continuous measurements could give a precise description of patient-specific iodine metabolism. Obviously, this goal cannot be achieved by currently available tools, therefore we hope that technology improvements will provide more advanced solutions at this regard. At present, such a large number of uptake measurements can be performed for research purposes only, and cannot become a routine practice in nuclear medicine. The effect of reducing the number of measurements without sacrificing accuracy has been successfully tested [34], but is beyond the scope of the present study. 
In the second place, we compared each of three widely used protocols $[4,28,29,35]$ as discussed in the previous sections - the EANM guidelines, ICRP 53 and a ME protocol, indicated by the Italian guidelines until 2012 - with OLINDA/EXM. The latter was chosen as gold standard because it has been FDA-approved for that purpose. As appears from the results of our study, some objections can be raised about the ICRP algorithms that do not use residence time, and $\mathrm{ME}$ dosimetric protocol, which excessively simplify patient kinetic. In fact, the ME dosimetric protocol overestimates the activities needed by more than 20\% (20\% in Graves' disease and $42 \%$ in ATN). Twenty percent represents the uncertainty level observed in our routine activity (unpublished data), and also the uncertainty level achievable by well-performed internal MIRD dosimetries, as reported in the literature [36]. Since this method can lead to higher uncertainties in the single patient even referred to the $A / D / m$ parameter (i.e. independently from mass estimation), this algorithm showed inadequate at least in patients with fast iodine metabolism, for whom the residence time estimated by the method does not represent the actual metabolism of these patients.

ICRP 53, on the other hand, relies on a fixed value as effective half-time and is only partially patient-dependent. Moreover, since the approach has been defined for radioprotection purposes, the underlying kinetic data are more representative of a healthy population. Although this publication is titled "Radiation Dose to Patients from Radiopharmaceuticals", ICRP 53 proposed data that do not describe successfully iodine kinetics in our patients. Compared to OLINDA, the ICRP 53 approach appears to underestimate required ${ }^{131}$ I activities, probably because of the difference in effective half-time values. Patients with small-pool syndrome have a short effective half-time: using a fixed effective half-time therefore results in an overestimation of residence time. Finally, it should also be considered that the six values for thyroid uptake $(5 \%, 15 \%, 25 \%, 35 \%, 45 \%, 55 \%)$ are only a partial representation of the wide range of possible uptake data actually measured, and almost all values required interpolation (if included in the range 5-55\%) or extrapolation (if higher than 55\%). Of the three approaches considered in this study, the EANM algorithm appears to provide the best dose estimation, since it yields very small differences (3\%) compared to OLINDA/EXM. For both methods (EANM and OLINDA/EXM), residence time estimation was mandatory and the bi-compartmental model was considered adequate for a correct determination of residence time $[10,11,31]$. Taking these results in due consideration, our routine practice is mostly oriented towards the EANM algorithm.

The third issue we addressed, was the use of a Unit-Density Sphere Model of OLINDA/EXM in activity estimation for ATN. A nodule shape is more similar to a sphere than to a thyroid, and the present dosimetric data seems to confirm this. In fact, in an ideal sphere the activity needed to deliver the target dose is lower than in an ideal ellipsoid; this is particularly important for small volumes, since the range of beta particles is comparable with the dimensions of the target object; therefore, the dependence of the absorbed dose to the geometry of the target increases while the volume reduces [32]. When ATN cases are considered, the activities computed with OLINDA are lower when using the sphere model compared to the thyroid model (data not presented). While the studied protocols apply the same algorithm to all benign thyroid diseases, independently from the specific pathology, the OLINDA/EXM software allows for a further activity personalization.

Finally, one further remark, the present work is essentially a dosimetric analysis. Our aim was to evaluate the best algorithm that could be used to calculate the amount of activity needed to reach a specific planned dose whenever OLINDA/EXM software is not available, whereas estimating the dose-outcome relationship was beyond the scope of this paper.

\section{Conclusion}

Compared to OLINDA/EXM, ICRP 53 approach and the ME dosimetric protocol (without estimation of residence time), although easier to be used, failed in accurately estimate activity in Graves' disease and ATN patients because of inadequate characterization of iodide metabolism.

The residence time seems to be well estimated by the bi-compartmental model $\left(R^{2}>0.90\right)$, although this topic was not deeply investigated in the present work. In absence of OLINDA/EXM software, EANM algorithm seems to allow an accurate calculation of the activity required to deliver a determined dose. Finally, The sphere model of OLINDA/EXM allows a further personalization on the activity calculation in ATN.

\section{Acknowledgements}

The authors would like to thank Dr Roberta Matheoud for her helpful suggestions and both Nuclear Medicine and Medical Physics staff for their support as well as Dr Katia Ansalone for her English editing.

\section{REFERENCES}

1. Lind P. Strategies of radioiodine therapy for Graves' disease. Eur $\mathrm{J}$ Nucl Med Mol Imaging 2002; 29 Suppl. 2: S453-S457.

2. Weetman AP. Radioiodine treatment for benign thyroid diseases. Clin Endocrinol (Oxf) 2007; 66: 757-764.

3. De Rooij A, Vandenbroucke JP, Smit JWA, Stokkel MPM, Dekkers OM. Clinical outcomes after estimated versus calculated activity of radioiodine for the treatment of hyperthyroidism: systematic review and meta-analysis. Eur J Endocrinol 2009; 161: 771-777.

4. Kobe C, Eschner W, Wild M et al. Radioiodine therapy of benign thyroid disorders: what are the effective thyroidal half-life and uptake of 131l? Nucl Med Commun 2010; 31: 201-205.

5. Van Isselt JW, de Klerk JMH, Lips CJM. Radioiodine treatment of hyperthyroidism: fixed or calculated doses; intelligent design or science? Eur J Nucl Med Mol Imaging 2007; 34: 1883-1884.

6. Leslie WD, Ward L, Salamon EA, Ludwig S, Rowe RC, Cowden EA. A randomized comparison of radioiodine doses in Graves' hyperthyroidism. J Clin Endocrinol Metab 2003; 88: 978-983.

7. Council Directive 97/43/Euratom, on health protection of individuals against the dangers of ionizing radiation in relation to medical exposure. [Internet]. OPOCE; 1997

8. International Commission on Radiation Units and Measurements (ICRU) Report n.67. 2002. Absorbed-dose Specification in Nuclear Medicine. Nuclear Technology Publishing. Ashford, England 2002.

9. Dottorini ME, Inglese E, Salvatori M, Signore A, Squatrito S VP. II Trattamento Radiometabolico Dell'ipertiroidismo. 2005.

10. Carlier T, Salaun P-Y, Cavarec M-B et al. Optimized radioiodine therapy for Graves' disease: two MIRD-based models for the computation of 
patient-specific therapeutic $131 \mathrm{I}$ activity. Nuclear medicine communications 2006: 559-66.

11. Hänscheid $\mathrm{H}$, Lassmann M, Reiners $C$. Dosimetry prior to I-131-therapy of benign thyroid disease. Z Med Phys 2011; 21: 250-257.

12. Peters $\mathrm{H}$, Fischer $\mathrm{C}$, Bogner $\mathrm{U}$, Reiners $\mathrm{C}$, Schleusener $\mathrm{H}$. Radioiodine therapy of Graves' hyperthyroidism: standard vs. calculated 131iodine activity. Results from a prospective, randomized, multicentre study. European journal of clinical investigation 1995: 186-193.

13. Howarth D, Epstein M, Lan L, Tan P, Booker J. Determination of the optimal minimum radioiodine dose in patients with Graves' disease: a clinical outcome study. Eur J Nucl Med 2001; 28: 1489-1495.

14. Canzi C, Zito F, Voltini F, Reschini E, Gerundini P. Verification of the agreement of two dosimetric methods with radioiodine therapy in hyperthyroid patients. Med Phys 2006; 33: 2860-2867.

15. Reschini E, Matheoud R, Canzi C et al. Dosimetry study in patients with autonomous thyroid nodule who are candidates for radioiodine therapy. J Nucl Med 1999; 40: 1928-1934.

16. Matheoud R, Canzi C, Reschini E, Zito F, Voltini F, Gerundini P. Tissue-specific dosimetry for radioiodine therapy of the autonomous thyroid nodule. Medical physics 2003: 791-798

17. Zanzonico PB. Internal radionuclide radiation dosimetry: a review of basic concepts and recent developments. J Nucl Med 2000; 41: 297-308.

18. Zanzonico PB, Becker D V, Hurley JR. Enhancement of radioiodine treatment of small-pool hyperthyroidism with antithyroid drugs: kinetics and dosimetry. J Nucl Med 2004; 45: 2102-2108

19. Stokkel MPM, Handkiewicz Junak D, Lassmann M, Dietlein M, Luster M. EANM procedure guidelines for therapy of benign thyroid disease. Eur J Nucl Med Mol Imaging 2010; 37: 2218-2228.

20. SnyderWS, Ford MR, Warner GGWSb. MIRD Pamphlet No.11 1975: "S, "absorbed dose per unit cumulated activity for selected radionuclides and organs. 1975.

21. Divoli A, Chiavassa S, Ferrer L, Barbet J, Flux GD, Bardiès M. Effect of patient morphology on dosimetric calculations for internal irradiation as assessed by comparisons of Monte Carlo versus conventional methodologies. J Nucl Med 2009; 50: 316-323.

22. Howard DM, Kearfott KJ, Wilderman SJ, Dewaraja YK. Comparison of I-131 radioimmunotherapy tumor dosimetry: unit density sphere model versus patient-specific Monte Carlo calculations. Cancer Biother Radiopharm 2011: 26: 615-621.
23. Senthamizhchelvan $\mathrm{S}$, Hobbs RF, Song $\mathrm{H}$ et al. Tumor dosimetry and response for $153 \mathrm{Sm}$-ethylenediamine tetramethylene phosphonic acid therapy of high-risk osteosarcoma. J Nucl Med 2012; 53: 215-224.

24. Stabin MG, Sparks RB, Crowe E. OLINDA/EXM: the second-generation personal computer software for internal dose assessment in nuclear medicine. J Nucl Med 2005; 46: 1023-1027.

25. Sgouros G, Frey E, Wahl R, He B, Prideaux A, Hobbs R. Three-dimensional imaging-based radiobiological dosimetry. Semin Nucl Med 2008; 38: 321-334.

26. ICRP. Radiation Dose to Patients from Radiopharmaceuticals. ICRP Publication 53. Ann. ICRP 18 (1-4), 1988

27. Hänscheid H, Canzi C, Eschner W et al. EANM Dosimetry Committee series on standard operational procedures for pre-therapeutic dosimetry II. Dosimetry prior to radioiodine therapy of benign thyroid diseases. Eur J Nucl Med Mol Imaging 2013; 40: 1126-1134

28. Panareo S, Rossi R, Fabbri S et al. A practical method for the estimation of therapeutic activity in the treatment of Graves' hyperthyroidism. Q J Nucl Med Mol Imaging 2011; 55: 576-585.

29. Schiavo M, Bagnara MC, Calamia I et al. A study of the efficacy of radioiodine therapy with individualized dosimetry in Graves' disease: need to retarget the radiation committed dose to the thyroid. J Endocrinol Invest 2011; 34: 201-205.

30. Allen $\mathrm{HC}$, goodwin WE. The scintillation counter as an instrument for in vivo determination of thyroid weight. Radiology 1952; 58: 68-79.

31. Rescigno A, Segre G. La cinetica dei farmaci e dei traccianti radioattivi: di Aldo Rescigno e Giorgio Segre [Internet]. 1961 [cited 2014 Jan 21].

32. Grosev D, Loncarić S, Huić D, Dodig D. Geometric models in dosimetry of thyroid remnant mass. Nuklearmedizin 2008; 47: 120-126.

33. Clerc J, Dagousset F, Izembart M et al. Radioiodine therapy of the autonomous thyroid nodule in patients with or without visible extranodular activity. J Nucl Med 1995; 36: 217-223.

34. Skanjeti A, Miranti A, Bianciotto D et al. How many measurements for good estimation of thyroid iodine metabolism: are three enough? Clin Transl Imaging 2013: 119

35. Kahraman D, Keller C, Schneider C et al. Development of hypothyroidism during long-term follow-up of patients with toxic nodular goitre after radioiodine therapy. Clin Endocrinol (Oxf) 2012; 76: 297-303.

36. Stabin MG. Uncertainties in internal dose calculations for radiopharmaceuticals. J Nucl Med 2008; 49: 853-860. 